CUM LAUDE

Revista del Doctorado en Derecho

Facultad de Derecho y Ciencias Sociales y Políticas UNNE

$\mathrm{N}^{\circ} 1$ - Diciembre 2014

Corrientes - Argentina

ISSN 2422-6408 (formato digital)

info@ revistacumlaude.com

FECHA DE RECEPCIÓN: 15/10/2014

FECHA DE ACEPTACIÓN: 12/11/2014

\title{
Pierre BourdieU Y La ViolenCia Simbólica
}

\author{
EdgaRdo Darío LóPEZ ViLlagRa \\ Facultad de Derecho, Ciencias Sociales y Políticas \\ UNIVERSIDAD NACIONAL DEL NORDESTE
}

\section{RESUMEN}

Según Pierre Bourdieu el objetivo de aprender o comprender una sociedad es entender cuáles son las estructuras de poder, entender cómo está conformado el ejercicio del poder para legitimar intereses de un grupo dominante sobre otro. Yacen en aquellas estructuras conflictos en donde los agentes pujan por la dominación de espacios sociales. De esta manera, la cultura se construye con un conjunto de mandatos, normas $\mathrm{y}$ valores que favorecen a algunos sectores en desmedro de otros.

\section{Palabras Clave}

Poder - dominación - género. 


\begin{abstract}
According to Pierre Bourdieu in order to understand a society is to understand what power structures are being made to understand how the exercise of power to legitimate interests of a dominant group over another. Conflicts lie in structures where agents fighting for dominance of social spaces. Thus, culture is constructed with a set of commands, norms and values that favor some sectors over others.
\end{abstract}

\title{
KEYWORDS
}

Power - domination - gender.

\section{ASPECTOS INTRODUCTORIOS}

En la actualidad, el interés de las Teorías de la Integración en Europa surge por el intento de aunar los conceptos sociológicos de acción y estructura. Sus autores parten del espectro de la sociología norteamericana y de las síntesis teóricas afianzadas sobre la década de los 90 del siglo XX, ya que toman como centro de su corpus teórico la integración de los niveles micro y macro del análisis social, pero terminan distanciándose de la misma corriente matizando muchos de sus postulados (Ritzer, 2001).

Se debe a Anthony Giddens la aportación de la Teoría de la Estructuración, evidenciando así la presencia de relaciones de correspondencia entre el sujeto y la estructura. A partir de esta tesis, Giddens se esfuerza por demostrar que las nociones de individuo y sociedad funcionan en un movimiento dialéctico enmarcado en un tiempo y 
espacio propios, cuyas interacciones auspician los procesos de cambio y de persistencia propios de la dinámica social (Fernández, 2004).

De acuerdo con este esquema, aparece la idea de estructuración como un factor dinámico de la reproducción de las prácticas, mediante el cual las estructuras cobran vigencia. Esto es, la dualidad entre sujeto y estructura produce una cierta estructuración en la que no existe una cosa sin la otra, alimentándose ambas en un continuum dinámico en el que los actores utilizan las propiedades estructurales para construir las relaciones sociales (Araujo González, 2003).

A través de la Teoría de la Estructuración, Giddens resalta las prácticas sociales como elementos derivados de la acción de los agentes humanos, que basan sus decisiones en función de los recursos que tienen a su disposición. Dichas prácticas sociales pertenecen de igual manera a todos los integrantes de la comunidad por su acumulación compartida en la familia, las amistades, el sistema de enseñanza y otros procesos de socialización. Así, recursos como el conocimiento formal del lenguaje, las reglas de cortesía, la identificación de los espacios comunes y otros, son utilizados regularmente en las rutinas sociales, reproduciendo con ello la permanencia de las estructuras.

Pero dentro de esta rutina de las prácticas sociales, los agentes actúan creativamente sobre las estructuras, imponiendo sus características individuales sobre el conocimiento compartido. Por consiguiente, las prácticas sociales reflejan las condiciones sociales heredadas, aunque las transforman gracias a las habilidades humanas individuales para conocer y reflexionar (Andrade Carreño, 1999).

Como señala Philippe Corcuff, existe en todo este mecanismo una visión circular de la construcción del mundo social, en la que la estructura se antepone a la 
acción y la condiciona a la vez que se refuerza en base a la acción del individuo. La estructura restringe, otorgando un marco de recursos que limita las posibilidades de conocimiento, pero son los mismos recursos estructurales los que facilitan al individuo obtener las facultades para las acciones y modificarlas (Corcuff, 2013). Cabe asimismo aclarar que el sujeto no goza de una capacidad de modificación ilimitada, pues los recursos se encuentran desigualmente distribuidos y, con esto, la acción humana también se circunscribe en razón del poder obrante entre los actores (Andrade Carreño, 1999).

Por otra parte, se debe a Jünger Habermas la vinculación entre la acción y la estructura organizada, en base a la expresión "la colonización del mundo de la vida" (Ritzer, 2001). La acción social se presenta como un espacio de tensión entre "el mundo del sistema y el mundo de la vida”. El primero, presentado como un ámbito integrado por las reglas de la organización social. El mundo de la vida en cambio, representa el sentido intersubjetivo e intencional de los individuos. Como consecuencia, la sociedad se consagra en una doble inmanencia definida por un lado por las acciones intencionales de los agentes, constreñidas por su interacción con un mundo del sistema (político, económico y social); y por el otro lado, por las estructuras o el mundo del sistema que se manifiestan como instancias de transformación social en base a las intencionalidades del mundo de la vida. Consecuentemente, los dos mundos deben comprenderse integrados e interactuando para que pueda existir la sociedad y la acción social (Giner, 2013). Como expresa Mario Heler (2007), citando a Jünger Habermas, estas interacciones son características de las sociedades modernas, en donde "el plexo de la vida social se reproduce a través de las 'acciones racionales con arreglo a fines' de sus miembros, controladas por medios generalizados de comunicación, y simultáneamente, 
a través de una voluntad común anclada en la práctica comunicativa de todos los individuos" (p. 93).

En el caso de Bourdieu, sus reflexiones se orientan como un esfuerzo por conciliar las Teorías Estructuralistas y las Subjetivistas en lo que él mismo ha enunciado como Estructuralismo Constructivista. Podemos destacar dos presupuestos esenciales que nos permiten comprender su teoría. Uno de ellos es que Bourdieu consigue combinar dos perspectivas que, históricamente, se han presentado por separado: el objetivismo (las estructuras exteriores al sujeto) y el subjetivismo (sus estructuras mentales). Otro aspecto fundamental es la noción de estructura como parte fundamental de sus disertaciones.

El mundo social para Bourdieu presenta estructuras objetivas, independientes de la conciencia y voluntad de sus agentes, y por lo tanto capaces de orientar sus prácticas y representaciones. Éste sería el sustento estructural de las acciones. Por otra parte, destaca Bourdieu, hablar de constructivismo sugiere que los esquemas de pensamiento, percepción y acción se revelan en base una cierta génesis social, lo que determina la adquisición de ciertos habitus que permanecen anclados a los espacios del campo social o grupos en los que el agente se desenvuelve (Bourdieu, 2000b). Es precisamente por la idea de esta génesis social de las acciones y esquemas de pensamiento de los actores propuesta por Bourdieu, por lo que su visión ha sido catalogada también como Estructuralismo Genético.

La base del Estructuralismo Constructivista de Bourdieu se sustenta en la posición epistemológica que debe asumir la Sociología como una ciencia relacional y disposicional. Como ciencia relacional, la Sociología adquiere el mandato de mostrar las realidades objetivas que no aparecen ante al científico por la simple observación de 
la realidad social, sino que exigen su aprehensión a través de la elaboración y validación con los métodos de la ciencia. Su carácter disposicional implica que el sociólogo debe situarse en consonancia con una filosofía de la acción que revele las potencialidades incorporadas en el cuerpo de los agentes, desplegadas en respuesta a las posiciones que ocupan en la estructura de las relaciones sociales (Bourdieu, 1997) ${ }^{1}$.

Al respecto, Alicia Gutiérrez señala en sus estudios sobre Bourdieu que con esta categorización el sociólogo retomó el modo de pensamiento relacional originado en la larga tradición estructuralista, identificando lo real con las relaciones; y oponiéndose a la perspectiva sustancialista que estudia el mundo social en referencia a las realidades que se revelan en la percepción directa: el individuo, el grupo, las interacciones. La reflexión en términos relacionales, prosigue Gutiérrez, conlleva situar las interacciones en un espacio y tiempo concreto, considerando las formas de la acción social que impregnan las representaciones que los agentes realizan sobre la estructura, en razón de sus posiciones y posibilidades (Gutiérrez, 2012).

Desde esta perspectiva sociológica, Bourdieu impone dos momentos de observación de las relaciones: el objetivista y el subjetivista. Las estructuras objetivas son percibidas por el sociólogo al apartar las representaciones subjetivas, revelando así las coacciones estructurales que condicionan aquellas representaciones (Bourdieu, 2000b). En un segundo momento, en el momento subjetivista, las representaciones subjetivas de los agentes sociales deben considerarse para comprender las luchas que transforman o preservan la estructura (Bourdieu, 2013; Ávila Penagos, 2002).

\footnotetext{
${ }^{1}$ La sociología supone, por su misma existencia, la superación de la oposición ficticia que subjetivistas y objetivistas hacen surgir arbitrariamente. Si la sociología es posible como ciencia objetiva, es porque existen relaciones exteriores, necesarias, independiente de las voluntades individuales y, si se quiere inconscientes (en el sentido de que no son objeto de la simple reflexión) que no puede ser captadas sino por los rodeos de la observación y de la experimentación objetivas (...). (Bourdieu, 1989, p. 18)
} 
El Estructuralismo Constructivista de Bourdieu basa sus principales presupuestos en las siguientes consideraciones:

- Las estructuras sociales externas al sujeto se presentan dentro de un espacio social general y de microespacios diferenciados: los campos sociales. Dentro del espacio social general se establecen las diferenciaciones entre las clases sociales como manifestaciones de la desigualdad en las posiciones de los grupos. Estas posiciones aparecen como resultado de la posesión de un cierto capital, englobando en este concepto de capital a todo recurso que devenido de la primacía que otorga su posesión, concede el poder de diferenciación y dominación entre los agentes y grupos de agentes, pudiendo resultar en bienes económicos, recursos culturales, sociales, simbólicos y tantos otros como se generen en el mundo social en un lugar y tiempo histórico determinado y determine luchas por su apropiación.

A su vez, siendo el mundo social un espacio compuesto por diferentes microespacios diferenciados llamados campos, cada relación de fuerza y de lucha por apropiarse de los capitales y posicionarse categorialmente entre los agentes y grupos, estará circunscrita a la posesión de los bienes específicos que se distribuyen en dicho microcampo. Asimismo, la posición en el espacio social general determina una apropiación de capital originario que permite acceder en mejor o peor posición a los capitales en juego dentro de cada microcampo.

- En razón de la relación dialéctica que se establece entre las estructuras objetivas y las subjetivas, la posición que llega a establecerse en el espacio general predispone al agente y genera determinadas disposiciones de acción o habitus. Los agentes interiorizan la lógica de las relaciones sociales y sus posiciones, y reproducen las prácticas que distinguen a las clases y las posicionan en sus microcampos: formas de 
pensar, el gusto, las necesidades estéticas, la forma de hacer las cosas, entre otras cuestiones. En consecuencia, el habitus preserva en su totalidad la reproducción del sistema de diferencias constituidas como orden social.

Corresponde a las Ciencias Sociales por tanto, descubrir los cimientos históricos y los determinantes sociales que se imponen como absolutos y eternos para desarticular la eficacia simbólica de la dominación (Bourdieu, 2013).

\section{VIOLENCIA SIMBÓLICA}

El concepto de violencia simbólica de Bourdieu representa un instrumento de análisis sociológico para explicar los medios y caminos de la dominación en diversos espacios sociales y culturales, sea que estas opresiones se revelen en el seno de grupos o de agentes sociales correspondientes a sociedades tradicionales, modernas, o entre las mismas naciones (Fernández, 2005; Dukuen, 2011).

La violencia simbólica representa "una forma particular de coacción” que opera a partir de distintas instancias legitimadoras a través de la imposición e incorporación compleja, paulatina e imperceptible de la arbitrariedad cultural (Fernández, 2005; Bourdieu, 2000a). Este conjunto de imposiciones redunda en la sumisión de los agentes sociales dominados a los dominantes, con la complicidad implícitamente consentida de los primeros, en virtud de la sumisión dóxica al orden consolidado (Bourdieu, 2012). En consecuencia, en la imposición de la violencia simbólica se exteriorizan las relaciones existentes entre las estructuras cognitivas y las estructuras objetivas que las producen (Bourdieu, 2013; Sandoval Robayo, 2002; Bourdieu y Passeron, 1996; Arango Gaviria, 2001; Silva Echeto y Browne Sartori, 2008). 
La noción de violencia simbólica comenzó a delimitarse claramente para Bourdieu a partir de los estudios sobre Sociología de la Educación realizados con Jean Claude Passeron y vertidos posteriormente en La reproducción. En estos estudios se categoriza "a toda acción pedagógica objetivamente como violencia simbólica" destinada a imponer una arbitrariedad cultural, siempre que por su forma de imponerse, por la estructura de lo que impone y por los grupos a quienes se impone se corresponda con los intereses objetivos de los grupos dominantes. La importancia de la acción pedagógica radica en que, a través de la misma, se asignan significaciones como legítimas, entendidas éstas como el modo de concebir las relaciones sociales. Estas asignaciones se ejecutan mediante una relación de comunicación desplegadas en tres instancias: a través de la educación difusa, que es aquella puesta en práctica por los miembros que comparten con el agente la misma formación social o grupo; de la educación familiar, por tanto, la deslizada en el seno del grupo familiar al que pertenece el agente; y especialmente, a través de la educación institucionalizada blindada por la escuela. Estas instancias colaboran objetiva e indirectamente en la creación de la doxa que perpetúa la dominación de las clases a partir de la inculcación de las significaciones en forma de saberes definidos para consolidar la reproducción de la dominación.

Christiane Chauviré y Oliver Fontaine, en El vocabulario de Bourdieu, postulan que los estudios en materia social de Bourdieu constituyen una opulenta edificación que da cimientos sólidos a una disciplina con vida propia: la sociología de la dominación y de la denuncia de la dominación. De esta manera la natural tensión que se establece en la lengua entre vocablos de signos opuestos, como lo sostiene el lingüista francés Saussure en su libro Curso de lingüística general, como se da en el caso preciso de dominantes/dominados organiza e instaura la compleja percepción de los aspectos más 
íntimos de la sociedad. El concepto de dominación sustituye en el pensamiento de Bourdieu al propio concepto generado por los autores marxistas de explotación. El sociólogo francés rechaza constantemente en sus estudios el exagerado economicismo proveniente de la Escuela Crítica, muy cercana al marxismo. Por otra parte, puede contrastarse y confrontarse esta percepción conceptual de Bourdieu con la propia definición que Michael Foucault da sobre el poder. Foucault circunscribe el concepto de poder a los complejos procesos de disciplinamiento de los cuerpos en el contexto social.

En otro sentido, el concepto de dominación es notablemente más dilatado que el de explotación, que únicamente aplica el autor de El capital a las relaciones de clases. Este concepto posibilita la generación de procesos dirigidos a revelar el sentido particular de la dominación masculina, la relación maestro/alumno en la escuela, jefe de servicio/empleados, entre los pares lingüísticos de tipo dialéctico. No cabe duda de que todo campo es espacio apropiado para la aparición de relaciones de dominación y de conflictos, como lo expresa el mismo paradigma del conflicto sustentado por el sociólogo alemán Ralf Dahrendorf. Es de resaltar, en esta relación dialéctica, que la dominación sería tan impracticable como inverosímil sin la obediencia dóxica de los dominados a los dominantes. Por otra parte cabe aclarar que de dicho sometimiento no se tiene auténtica conciencia, sin embargo los sometidos pueden generar tácticas y destrezas que posibiliten mecanismos de necesaria resistencia canalizados a través de distintos mecanismos de disputas y combates. El pensamiento bourdieusiano de lo social es integralmente una introspección constante que genera una especulación en torno a juicios dirigidos a describir y contextualizar el fenómeno de la dominación.

En cuanto a la cuestión de la ideología dominante, Bourdieu publica junto con Luc Boltanski un libro que se denomina La producción de la ideología dominante, en el 
año 2009, donde se hacen valiosas precisiones sobre los mecanismos sociales, políticos y económicos que dan vida al conjunto de preceptos y valores que sustentan a la ideología que profesan los que dominan (Bourdieu y Boltanski, 2009).

En esta estructura de depuración teórica y conceptual emerge el fenómeno de la dominación masculina. Aunque existan apariencias de que el sostenimiento de dicha relación de dominación está marcado equívocamente por la naturaleza biológica, no constituye otro ejemplo que una derivación del concepto de dominación que el sociólogo francés construye a los efectos de la interpretación, comprensión y explicación de la realidad social.

En conclusión, ninguna dominación se desplegaría factiblemente si en su conjunto, los mismos dominados no colaboraran para su sometimiento. Se destaca como ejemplo el caso paradigmático de dominación simbólica. En este sentido se puede hacer mención a la sumisión femenina como elemento necesario para el establecimiento de las eficaces relaciones de dominación en una sociedad de aspectos fuertemente patriarcales (Chauviré y Fontaine, 2008).

La eficacia de la acción pedagógica es conmensurable por su capacidad de generar "habitus duraderos, transferibles y exhaustivos" y, con ello, plausibles de reproducir a través de las prácticas los principios de arbitrariedad cultural de la clase o grupo dominante. La acción pedagógica sustituye a la coacción física, imponiéndose de manera sutil sobre las estructuras cognitivas y las disposiciones de los agentes. Esta característica la torna más efectiva, pues su interiorización voluntaria pero no consciente produce un mayor desconocimiento en los agentes de ser depositarios de la arbitrariedad cultural (Ávila Penagos, 2002; León Cáceres, 2005). Por estos motivos son por los que Bourdieu describe a la violencia simbólica como aquella "ejercida sobre un agente 
social con su complicidad” (Bourdieu y Passeron, 1996). El desconocimiento del agente parte de un estado pre-reflexivo, generado por la aceptación dóxica del mundo social y de sus supuestos como resultado de la construcción de sus estructuras cognitivas a través de las mismas estructuras objetivas de la sociedad (Bourdieu y Wacquant, 2005).

La violencia simbólica es para Bourdieu un tipo más de violencia que coexiste con otras especies como la violencia física o económica. Sin embargo, su poder de imposición es mucho más feroz y agresivo que las coacciones directas, precisamente por la sutileza de su incorporación institucionalizada. En consecuencia, su imposición se disgrega dentro de todos los campos sociales, permitiendo reconocer cómo se ejerce y, especialmente, cómo se reproduce el poder simbólico originado dentro de ellos (Campos Santelices, 2010; Levaggi, 2007; Bourdieu, 1985).

Por estos motivos son por los que el sociólogo francés resalta, en Razones prácticas. Sobre la teoría de la acción, la característica de la teoría de la violencia simbólica como una derivación de las teorías de la producción de la creencia, entendiendo a la creencia como una adhesión inmediata a las conminaciones del mundo nacida de la sintonía entre las estructuras cognoscitivas y las estructuras de la dominación (Foucault, 1991; Vázquez, 1999; Gutiérrez, 2003). Esta consustanciación entre las estructuras objetivas y las percepciones de los agentes manifestada en forma de disposiciones y consolidada en la creencia, permite que quienes se someten a un acto de imposición perciban que deben obedecer sin plantearse los fundamentos de la obediencia. Como consecuencia, la violencia simbólica transmuta las relaciones de dominación en relaciones afectivas, y con ello, decanta en un mayor acrecentamiento del capital o reconocimiento simbólico para ciertos grupos o agentes sociales. El capital simbólico que se genera como resultado de esta transmutación -explica Bourdieu- es 
común a todos los integrantes de un grupo, y se manifiesta en la relación con los agentes de un grupo que poseen ciertas propiedades (arriba/abajo, masculino/femenino, grande/pequeño) y en la observación de la percepción de los otros agentes de aquellas propiedades; lo que consiente el establecimiento de categorías sociales (los de arriba/los de abajo, masculino/femenino, los grandes/los pequeños). Estas categorías sociales generan estrategias colectivas e individuales de unión y separación entre grupos, familias, clanes y tribus (como los matrimonios y coaliciones, o por el contrario, el tabú de la inmediación con el contario o el casamiento con persona inferior) a fin de conservar o aumentar el capital simbólico en la unión con los grupos que los poseen, o de separase de aquellos grupos que no lo poseen o lo tienen en menor medida (Bourdieu, 1997).

No podemos dejar de hacer mención, dentro de la teoría de la violencia simbólica, a la relevante relación que destaca Bourdieu de la misma con la dominación masculina, por constituir el ejemplo más persistente en la historia de las relaciones sociales de la imposición de la violencia simbólica (Gudiño Bessone, 2011). En la coacción que supone la estructuración de un orden sexual en el contexto de una social androcéntrica, es donde se manifiesta por excelencia la violencia invisible, amortiguada, insensible y por tanto simbólica, que legitima la arbitrariedad de las relaciones de dominación bajo el fundamento de las diferencias biológicas (Bourdieu, 2000a; Sandoval Robayo, 2002; Palma, 1992; Fernández Poncela, 2008).

Bourdieu remarca cómo ciertos hechos sociales, como la división sexual del trabajo, los espacios, momentos e instrumentos reservados a los hombres o mujeres, se han fundamentado desde una perspectiva biológica que, resaltando el cuerpo y su diferenciación anatómica, justifican en la naturaleza las diferencias sociales entre lo 
masculino y femenino (Irigaray, 2009; Turner, 1989). Es así que el primer centro de la dominación masculina se expresa en la definición social de los órganos sexuales, en donde el principio masculino aparece como la medida de todo, como se manifiesta en la clásica concepción de la vagina como falo invertido (Fernández Poncela, 2000; Mauss, 1971). Así, el mismo acto sexual se concibe desde la lógica androcéntrica como un acto de posesión, apropiación y dominación (Bourdieu, 2000a; Moi, 2011; Gamero Cabrera, 2012; Córdova Plaza, 2003; Vázquez García, 2002). El otro centro de la dominación masculina reside para Bourdieu en el "mercado de los bienes simbólicos", esto es, en la consideración histórica de la mujer como un objeto intercambiable para el mercado matrimonial, con una existencia limitada por y para la mirada de los demás, y de donde se desprenden expectativas de conducta y acción como la simpatía, la docilidad y la sumisión (Dri, 2003; Blanco, 2009). Esta naturalización de las relaciones de dominación masculina, al igual que todas las relaciones de dominación mediadas por la violencia simbólica, genera esquemas de percepción y apreciación espontáneos en los agentes, en este caso las mujeres, que los hace propensos a actuar conforme a dichas relaciones (Bourdieu, 2000; Sandoval Robayo, 2002; Fowler, 2001).

La fuerza de la dominación masculina operada mediante la noción de lo natural, tiene dos efectos principales: diferenciar la posición de los agentes acorde al orden sexual, lo que produce determinadas 'inclinaciones' en cada uno de ellos, y establecer el marco diferenciador capaz de asentar las diferencias de las posiciones (Mathieu, 1985). En este proceso de naturalización colaboran de manera manifiesta tres instancias: la familia, que brinda la experiencia precoz de la división sexual del trabajo y su legitimación; la Iglesia, signada por un profundo antifeminismo; y la escuela, a través de sus estructuras jerárquicas con connotaciones sexuales delineadas a través de una 
representación patriarcal de las relaciones (Bourdieu, 2000a; Butler, 2007; Chiarotti, 2004; Lamas, 2000).

\section{CONCLUSIONES}

La distribución desigual del poder entre hombres y mujeres da vida al sistema patriarcal. Fueron numerosos los autores que desde las ciencias sociales intentaron desmantelar este sistema de convivencia. De esta manera, autores como Sartre, Simone de Beauvoir, y hasta el mismo Bourdieu, formaron parte de una línea de pensamiento que estuvo enfocada en subvertir las bases mismas de un sistema de dominación social que parecía obsoleto desde muchos puntos de vista. De esta manera, en la década del 60 se vivió con gran efervescencia las luchas por los derechos de la mujer en fortalecimiento de las luchas de los sectores feministas. No caben dudas que Bourdieu representó, como intelectual comprometido, un importante emblema para aquellas luchas. Fue el Mayo Francés el que desató las fuerzas contenidas dentro del seno de la sociedad europea, y con vastas consecuencias en el resto de Occidente. El concepto de violencia simbólica que Bourdieu construye a lo largo de sus investigaciones, desde aquellos estudios sobre la sociedad cabilla, plasmados en su obra La dominación masculina junto con el estudio que hace del sistema educativo francés en el célebre texto Los herederos conforma el núcleo conceptual de aquel aporte. La superación de las diferencias sexuales para abrir paso al planteamiento de las diferencias culturales, ha sido uno de los grandes aportes de Pierre Bourdieu no sólo a las ciencias sociales, sino también a la instrumentación de políticas de igualdad de género, a través de las cuales se ha pretendido construir un paradigma distinto. 
La obra de Bourdieu en este sentido ha sido determinante para la visualización de los esquemas de poder a través de los cuales los distintos agentes se enfrentaban en el campo social. La superación del concepto marxista de explotación y la apertura hacia una nueva dimensión en donde el monopolio de la dominación encuentra su eje temático, también es producto de aquellas interpretaciones que Bourdieu fue bosquejando a lo largo de su vida académica. Es por ello que en su preocupación por la educación y el papel de la mujer en la sociedad contemporánea debe buscarse la construcción conceptual de la violencia simbólica en un todo más complejo que constituye la inmensidad del legado de Bourdieu a través de sus numerosas publicaciones.

Finalmente, revelar los mecanismos intrínsecos que operan en el corazón de las sociedades a través de la cultura, constituyó una inspiración constante de las cuales la violencia simbólica parece ser uno de los resultados más fructíferos, aplicado inclusive a otras áreas en donde el conflicto es la forma en que los actores se interrelacionan en el espacio social.

\section{REFERENCIAS BIBLIOGRÁFICAS}

Andrade Carreño, A. (1999). La fundamentación del núcleo conceptual de la teoría de la estructuración de Anthony Giddens. Revista Sociológica, 14(40), 135-138.

Arango Gaviria, L. G. (2001). Democratización de las relaciones de género y nuevas formas de dominación de clase en América Latina: reflexiones a partir del caso colombiano. Revista Colombiana de Sociología, 6(2). 
Araujo González, J. (2003). El concepto de fiabilidad en Anthony Giddens. Análisis y crítica de una alternativa en la teoría sociológica. México: Plaza Valdez Editores.

Ávila Penagos, R. (2002). Las relaciones entre la educación y la cultura en Pierre Bourdieu. Revista Colombiana de Sociología, 7(1), 11-14.

Blanco, J. (2009). Rostros visibles de la violencia invisible. Violencia simbólica que sostiene el patriarcado. Revista Venezolana de Estudios de la Mujer, 14, 32.

Bourdieu, P. (1985). Qué significa hablar. Madrid: Akal.

Bourdieu, P. (1989). La fotografía, un arte intermedio. México: Nueva Imagen.

Bourdieu, P. (1997). Razones Prácticas. Sobre la Teoría de la Acción. Barcelona: Editorial Anagrama.

Bourdieu, P. (2000a), La dominación masculina. Barcelona: Anagrama.

Bourdieu, P. (2000b). Cosas dichas. Barcelona: Gedisa Editorial.

Bourdieu, P. (2012). Violence simbolic. Revista Latina de Sociología, (2).

Bourdieu, P. (2013). La nobleza de Estado. Educación de élite y espíritu de cuerpo. Buenos Aires: Siglo Veintiuno Editores.

Bourdieu, P. y Boltanski, L. (2009). La producción de la ideología dominante. Buenos Aires: Ediciones Nueva Visión.

Bourdieu, P. y Passeron, J. C. (1996). La reproducción. Elementos para una teoría del sistema de enseñanza. México: Distribuciones Fontamara S.A.

Bourdieu, P. y Wacquant, L. (2005). Una invitación a la sociología reflexiva. Buenos Aires: Siglo Veintiuno Editores.

Butler, J. (2007). El género en disputa. Barcelona: Paidós.

Campos Santelices, A. (2010). Violencia Social. San José, Costa Rica: Euned. 
Chauviré, C. y Fontaine, O. (2008). El vocabulario de Bourdieu. Buenos Aires: Atuel.

Chiarotti, S. (2004). Nacimiento del Estado laico y el proceso de separación del Estado con la Iglesia. En VV.AA. Fundamentalismos. Las mujeres latinoamericanas se organizan. Córdoba: Católicas por el Derecho a Decidir.

Corcuff, P. (2013). Las nuevas Sociologías. Principales corrientes y debates, 19802010. Buenos Aires: Siglo Veintiuno Editores.

Córdova Plaza, R. (2003). El concepto de habitus de Pierre Bourdieu y su aplicación a los estudios de género. Colección Pedagógica Universitaria, (40), 1-10.

Dri, R. (2003). Símbolos religiosos en la construcción de la identidad popular. En R. Dri (Comp.), Símbolos y fetiches religiosos en la construcción de la identidad popular (vol. 2, pp. 13-26). Buenos Aires: Biblos.

Dukuen, J. (2011). Retomando un debate: la teoría de la violencia simbólica en Bourdieu y la noción de ideología en Althusser, frente al problema de la reproducción. Intersticios, 5(1).

Fernández, J. M. (2005). La noción de violencia simbólica en la obra de Pierre Bourdieu: una aproximación crítica. Cuadernos de Trabajo Social, 18, 7-31.

Fernández Poncela, A. M. (2000). Protagonismo femenino en cuentos y leyendas de México y centroamérica. Madrid: Narcea, S.A. de Ediciones.

Fernández Poncela, A. M. (2008). Discursos sociales tradicionales: estrategias disciplinarias y temores. Tramas, 30, 213-244.

Fernández, M. (2004). Nombres del Pensamiento Social: miradas contemporáneas sobre el mundo que viene. Buenos Aires: Ediciones del Signo.

Foucault, M. (1991). Microfísica del poder. Madrid: Ediciones de La Piqueta. 
Fowler, B. (2001). La dominación masculina en la obra de Pierre Bourdieu. Guaraguao, Revista de Cultura Latinoamericana, 5(13), 64-87.

Gamero Cabrera, I. G. (2012). Los efectos de la dominación simbólica en el feminismo", Revista internacional de filosofía, (13), 189-200.

Giner, S. (Coord.), Teoría Sociológica Moderna. Barcelona: Editorial Planeta S.A.

Gudiño Bessone, P. (2011). ¡Dios está con nosotras! La disputa simbólica por la sexualidad y la reproducción en el catolicismo. Prácticas de oficio. Investigación y reflexión en Ciencias Sociales, (7-8).

Gutiérrez, A. (2012). Las prácticas sociales. Una introducción a Pierre Bourdieu. Córdoba: Eduvim.

Gutiérrez, A. B. (2003). Con Marx y contra Marx: El materialismo en Pierre Bourdieu. Revista Complutense de Educación, 14(2).

Heler, M. (2007). Jurgen Habermas y el proyecto moderno. Cuestiones de la perspectiva universalista. Buenos Aires: Biblos.

Irigaray, L. (2009). Ese sexo que no es uno. Madrid: Akal.

Lamas, M. (2000). Diferencias de sexo, género y diferencia sexual. Cuicuilco, 7(18), 124.

León Cáceres, P. C. (2005). La violencia simbólica en la revuelta estudiantil. El movimiento estudiantil de Antropología y Sociología en la Universidad de Caldas. Revista Colombiana de Sociología, (24).

Levaggi, C. (2007). La Virgen de San Nicolás “¿Símbolo popular posmoderno?”. En R. Dri (Comp.), Símbolos y fetiches religiosos en la construcción de la identidad popular (vol. 2, pp. 45-70). Buenos Aires: Editorial Biblos. 
Mathieu, N. (1985). L'arraisonnement des femmes, essais en anthropologie des sexes. París: Editions de l'Ecole des hautes études en Sciences Sociales.

Mauss, M. (1971). Técnicas y movimientos corporales. En M. Mauss y C. Lévi-Strauss (Coords.), Sociología y antropología. Madrid: Tecnos.

Moi, T. (2011). Apropiarse de Bourdieu: la teoría feminista y la sociología de la cultura de Pierre Bourdieu. El feminismo como critique. Feminaria, 14(26-27), 1-20.

Palma, M. (1992). La mujer es puro cuento. Feminidad aborigen y mestiza. Bogotá: Tercer Mundo/Indigo.

Ritzer, G. (2001). Teoría Sociológica Clásica. España: Mc Graw Hill.

Sandoval Robayo, M. L. (2002). Pierre Bourdieu y la teoría sobre la dominación masculina. Revista Colombiana De Sociología, 7.

Silva Echeto, V. M. y Browne Sartori, R. (2008). Comunicación, violencia y poder simbólico en la sociología de Pierre Bourdieu. Nómadas, (17).

Turner, B. (1989). El cuerpo y la sociedad: exploraciones en teoría social. México: Fondo de Cultura Económica.

Vázquez García, F. (2002). Pierre Bourdieu: la sociología como crítica de la razón. España: Editorial Montesinos.

Vázquez, F. (1999). Historicidad de la razón y teoría social: entre Foucault y Bourdieu. Revista Mexicana de Sociología, (2), 189-212.

\section{Curriculum vitae}

El autor es Abogado egresado de la Facultad de Derecho y Ciencias Sociales y Políticas de la Universidad Nacional del Nordeste. Doctor en Historia de América, Facultad de Geografía e Historia, Departamento de Historia de América, Universidad de 
Sevilla, Reino de España, 2012, Programa de Doctorado "Historia, Literatura y Poder: Procesos interétnicos y culturales en América", con Mención de Calidad (MCD -2006 00139), Acreditación Real Decreto 1339/2007. Máster en Historia de América, Universidad Internacional de Andalucía, Huelva España, Tesis aprobada con sobresaliente Cum Lauden, Mayo de 2009. Diploma de Estudios Avanzados (DEA) en Historia de América. Universidad de Sevilla. España. 24 de junio de 2009. Tesis aprobada con sobresaliente cum lauden. Profesor Titular por concurso de Sociología Cátedra "A"; Profesor Adjunto por concurso de Historia Constitucional Argentina Cátedra "A" de la Facultad de Derecho y Ciencias Sociales y Políticas, Universidad Nacional del Nordeste. Investigador categorizado en el programa de incentivos docentes. Coordinador e integrante de proyectos de investigación. Becario de la Fundación Carolina entre 2006 y 2011. Director becarios de investigación de pregrado y tesistas doctorales. Autor de libros, capítulos de libros y artículos científicos en revistas nacionales e internacionales. Correo electrónico: dario.lovi@ hotmail.com 